\title{
THE INFRARED AND X-RAY CONTINUA OF QUASARS: IS THERE A CONNECTION?
}

\author{
Martin Elvis, Jonathan McDowell and Belinda J. Wilkes \\ Harvard-Smithsonian Center for Astrophysics \\ Cambridge, Massachusetts, USA.
}

The first Einstein IPC X-ray spectra of quasars had slopes similar to their infrared spectra (Elvis et al. 1986) suggesting that the infrared and X-ray continua of quasars might be two ends of a single power-law extending over at least 4 decades of the electromagnetic spectrum. In retrospect this was a bold claim based as it was on only eight quasars with limited infrared coverage. If true though it would greatly simplify the structure of the quasar continuum.

We have been collecting data to test this hypothesis. We have made ground-based 1$10 \mu \mathrm{m}$ observations and have extracted co-added IRAS data for almost all the 33 quasars in the large Einstein IPC spectral study of quasars by Wilkes and Elvis (1987). We have also extended the $x$-ray energy range through the use of MPC (2-10kev) data. Much useful data was also taken from the literature, particularly Neugebauer et al. (1987). With this improved data set we have made Quasar Energy Distributions for all the objects. Sometimes the 'single IR-to-X-ray power-law' fits extraordinarily well (fig. 1 ).

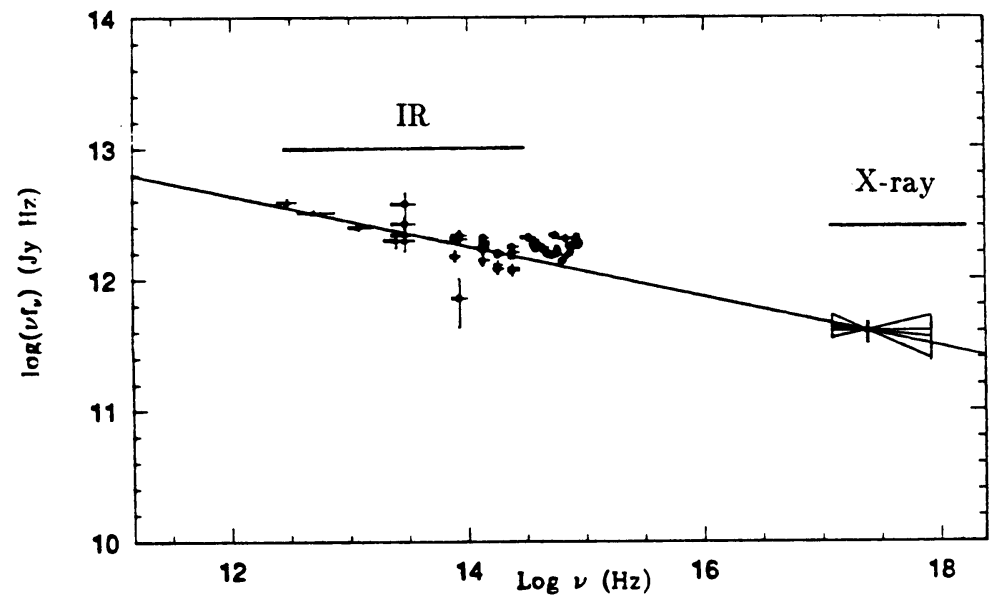

figure 1: Quasar Energy Distribution from IR to X-ray for PG1613+658 (MKN 876)

Generally though the results show a somewhat more complex picture. The infrared continua are rarely pure power-laws and need either long wavelength cut-offs and/or 'thermal' bumps to describe them adequately. Carleton et al. (1987), Ward et al. (1987) and Edelson and Malkan (1986) have explored these possibilities in Seyfert galaxies. At this point we have not modeled the infrared carefully or looked at alternatives to the 'single IR-to-X-ray power-law' hypothesis. We have instead asked the simpler question 
"What fraction of our objects fit the hypothesis and to what degree?". Table 1 lists the results.

Table 1: Degree of consistency with 'single IR-X-ray power-law' hypothesis

\begin{tabular}{|c|c|c|c|}
\hline & IR continuum type & Radio-quiet & Radio-loud \\
\hline & Pure power-law & 31 & 21 \\
\hline \multirow[t]{2}{*}{ Consistent } & $\{$ Cut-off power-law & 11 & 2 \\
\hline & Lump+cut-off power-law & 4 & $3 \mathrm{~J}$ \\
\hline \multirow[t]{3}{*}{ Inconsistent } & $\{\mathrm{X}$-ray slope too flat & $2\}$ & $8\}$ \\
\hline & Peculiar & $4 J$ & \\
\hline & Fraction consistent & $11 / 17$ & $7 / 15$ \\
\hline
\end{tabular}

Wilkes and Elvis (1987) found that only radio-quiet quasars had the steeper slopes needed to join to the infrared, and indeed radio-loud objects clearly do less well in Table 1. In the radio-quiet cases we can only clearly rule out the 'single IR-to-X-ray power-law' hypothesis in 2 cases. Four others are peculiar and only fit if unlikely assumptions are made. The remaining 11 quasars fit easily if cut-offs in the power-law at $\sim 25-100 \mu \mathrm{m}$ are allowed. 8 of these 11 also require small 'thermal' bumps at $\sim 10-20 \mu \mathrm{m}$, as in Seyferts (Carleton et al. ). In all 11 cases the $\mathrm{x}$-ray slope is consistent with the infrared-to- $X$-ray slope. The $\mathrm{x}$-ray spectra are oversimplified since they are allowed only a single slope, although they span nearly 2 decades. The real spectra certainly have a some curvature (Wilkes and Elvis 1987).

We beleive that the original hypothesis has survived the test with only reasonably small modifications. This is still only a consistency check. The signal-to-noise and energy resolution of the infrared and X-ray data is insufficient to make strong statements. Another test is to measure the infrared spectra for particularly ' $x$-ray quiet' quasars since they should have systematically steeper infrared slopes. We are now pursuing this. The idea of a single infrared to $x$-ray power-law is appealing as a way of conceiving the overall quasar continuum. While it is not proven, and may yet not be true, it is a challenging concept for theoretical and observational investigation.

The Quasar Energy Distributions project is the work of many people. We particularly thank S. Willner, K. Arnaud, R. Green, J. Bechtold, E. Polomski, S. Oey, and J.Lockman. This work is supported by the NASA ADP program through grant NAG8-689, and through NASA contract NAS8-30751.

\section{REFERENCES}

Carleton N.P. et al. , 1987, Ap. J., 318, 595.

Edelson R.A.and Malkan M., 1986, Ap. J., 308, 59.

Elvis M. et al. , 1986, Ap. J., 310, 291.

Neugebauer G. et al. , 1987, Ap. J. Suppl., 63, 615.

Ward M.J. et al. , 1987, Ap. J., 315, 74.

Wilkes B.J. and Elvis M., 1987, Ap. J., 323, 243. 


\section{DISCUSSION}

WILLS The mid-IR spectra show peaks and curvature (e.g., Neugebauer, Soifer, and Miley 1985, Ap. J., 295, L27) and the near IR spectra of (non-blazar) quasars almost always show a $3 \mu \mathrm{m}$ bump (Wills 11987, IAU Symposium No. 124, "Observational Comology," ed. Burbidge, Hewitt, and Fang) so it is not valid to describe the IR spectra by a power law, and not meaningful to extrapolate this spectral slope to $x$-ray frequencies.

ELVIS There certainly are deviations from a simple power-law and the mysterious " $3 \mu \mathrm{m}$ bump" is one of these. Indeed many AGNs contain some dust. However, Carleton et al. (1987) showed that the idea of an underlying baseline power-law gives an extraordinarily tight correlation with $X$-ray emission. In quasars where the UV and $X$-ray continua do not appear to be reddened this power-law dominates. In this kind of object (which includes most PG quasars), it does seem reasonable to us to try to connect the $X$-ray and IR bands.

BIERMANN Chini, Kreysa, and I have observed all radio-weak $A G N s$ from Neugebauer's list at $1.3 \mathrm{~mm}$ with the $30-\mathrm{m} \mathrm{mm}$-telescope on Pico Veleta (Spain). All objects have $1.3 \mathrm{~mm}$ flux densities of a few $\mathrm{mJy}$ or less, suggesting that the FIR-emission is kpc-scale dust, heated by the active nucleus.

ELVIS These are crucial observations. I look forward to studying your preprint. We hope to use JCMT at $0.8 \mathrm{~mm}$ to set similar, perhaps more restrictive, limits on quasars with no sign of reddening in the UV and/or X-ray bands.

WORRALL There is evidence that the effective spectral index between the infrared (or optical) and $X$-ray bands steepens with increasing source luminosity. Does your infrared data suggest any steepening of an underlying power law with source luminosity?

ELVIS Our prime sample covers only a small range of $\alpha_{o x}$ and $\mathrm{X}$-ray luminosity. We are now collecting data to look at steep $\alpha_{o x}$ quasars for this effect. Most of these large $\alpha_{o x}$ objects will also have large $L_{X}$. 